\title{
A SILICON HEAT EXCHANGER WITH INTEGRATED INTRINSIC-POINT HEATER DEMONSTRATED IN A MICROPROPULSION APPLICATION
}

\author{
Robert L. Bayt \\ United Technologies Research Center \\ E. Hartford, Connecticut 06108 \\ Kenneth S. Breuer \\ Division of Engineering, Brown University \\ Providence, Rhode Island 02912
}

\begin{abstract}
Efficient heating of a fluid is demonstrated using a novel heat exchanger in which bulk silicon forms both the heater structure and the resistive heating elements. Current passed through the heater raises the temperature of the heater fins and this energy is transferred to a fluid flowing between adjacent fins. By exploiting the change in sign of the temperature coefficient of resistivity of the heavily doped silicon, the temperature of the system is stably maintained at the intrinsic point. A heat exchanger of this nature is integrated with a nozzle, resulting in a microthruster with elevated chamber temperature, which greally improves the specific impulse, or thrust per unit weight flow of propellant. A numerical model is presented to optimize the heater design. Benchtop tests demonstrate the inherent stability of the intrinsic point heater design while thrust tests demonstrate the improved fuel economy of the micropropulsion system.
\end{abstract}

\section{INTRODUCTION}

A wide variety of MEMS applications employ an integrated heater, including microreactors (to catalyze reactions) and valves (to actuate the poppet). In general, these heaters typically use a thin-film metal or polysilicon as a resistor to generate heat. These systems work well, but are typically hampered by the relatively low contact area between the fluid and the heating element [1]. The heater effectiveness can be characterized as the ratio of the observed temperature rise of the fluid to the maximum possible temperature rise that could occur for a given wall temperature:

$$
\varepsilon=\frac{T_{\text {outlet, fluid }}-T_{\text {inlet }, \text { fluid }}}{T_{\text {outlet }, \text { wall }}-T_{\text {inles, fluid }}}
$$

Thus, a heater with very good thermal contact between the fluid and heating element would bring the fluid up to the wall temperature and achieve an effectiveness of unity. Since the dominant heat transfer mechanism is forced convection, heat transfer to the fluid is governed by Newton's Law of cooling,

$$
q=h A\left(T_{\text {fluid }}-T_{\text {wall }}\right),
$$

where the area of fluid-heater interaction $(A)$ is often the practical limitation for achieving high effectiveness.

A second issue of common concern in gas flow heat exchangers is heater stability. This arises because the gas viscosity increases with temperature. Thus, if a local hotspot develops, the fluid viscosity will increase, increasing the pressure drop across the heat exchanger passage and thus reducing the mass flow through the channel. This, in-turn, reduces the cooling and further raises the temperature in the channel. In this manner a thermal runaway can ensue leading to device failure.

This paper presents a microfabricated solution for achieving a highly effective heater in which the heater elements are formed from the structural material of the fluid system - single crystal silicon. This architecture has two principal advantages. First, the electrical and mechanical functions are combined. Second, the thermo-electric properties of silicon allows for an inherently stable operation at high temperature without risk of thermal runaway.

In this paper, the heater is used to increase the chamber temperature for fluid entering a micronozzle to create a propulsion system for a microspacecraft. In such applications, maximizing the thrust per unit weight of propellant (i.e. specific impulse, $I_{s p}$ ) is highly desirable and is enhanced by increasing the chamber temperature by preheating the gas prior to expansion. We also present a simple model to maximize the heater effectiveness while minimizing the pressure drop through the fin array. Varying the number of passages, their length, thickness and spacing accomplishes this goal.

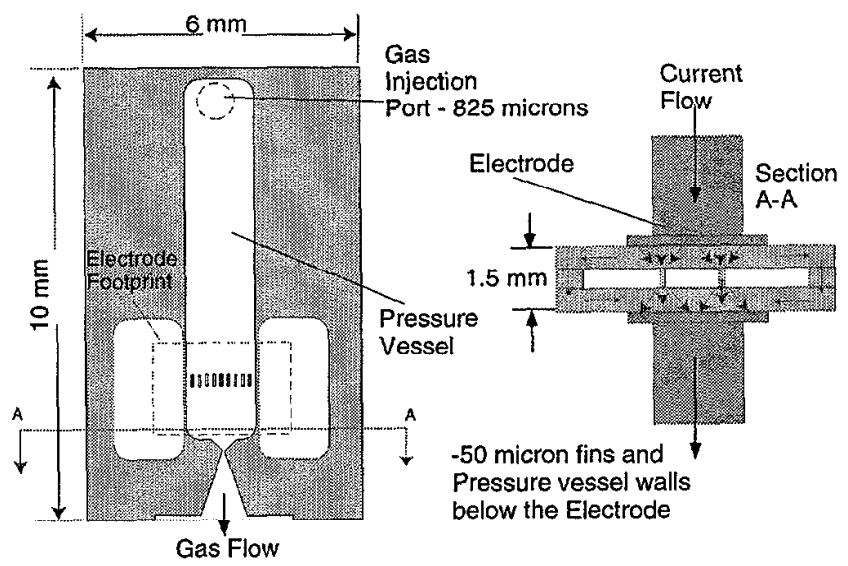

Figure 1. Schematic of device. The thruster is deep etched to provide maximum surface area of the heat exchanger and a highaspect ratio nozzle.

\section{HEATER OPERATION}

The overall heater design is shown in Figure 1. Gas enters the port in the top wafer and flows through the heat exchanger, which is defined by narrow parallel fins upstream of the nozzle. The entire structure is fabricated from heavily doped P-type silicon. An electric current flows from top to bottom through the device, and is focused through the fins, heating them resistively.

The properties of silicon as a semiconductor are used to maintain stable operation of the device at high temperature. By fabricating the heater using heavily doped P-type silicon wafers, the dopant holes will be the primary charge carriers at lowtemperatures. As power is dissipated and the temperature of the device increases, the electrons bound in the in the silicon valence bands become thermally excited to the conduction band. When the number of intrinsic carriers becomes dominant, the resistivity of 
the material is governed by statistical mechanics, and decreases exponentially with temperature.

This trend in resistance is shown in figure 2 , and indicates the intrinsic point for a silicon sample doped to $10^{19}$ atoms/cc [3]. The key feature of figure 2 is the change in the sign of the temperature coefficient of resistivity at the intrinsic point.

The heater is operated in a constant current mode. As the current increases, the dissipated power, temperature, and resistance, all rise in response. However, when the intrinsic temperature is reached, any increase in dissipated power and temperature results in a drop in resistance. Consequently the dissipated power falls and the device returns to operation at the intrinsic point. Thus, a stable operating temperature is maintained with the feedback provided by the resistive properties of silicon.

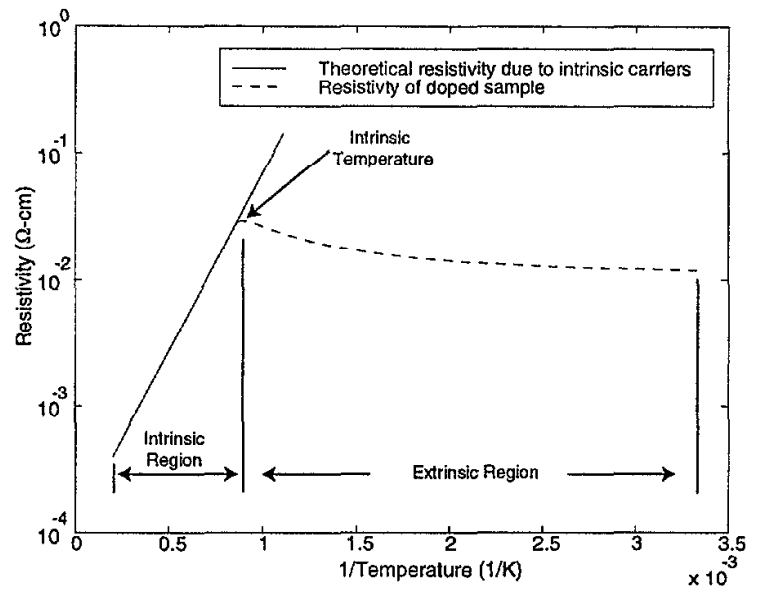

Figure 2. The resistivity of doped silicon is peak when intrinsic and dopant charge carriers are equal. This plot is for a P-type $10^{19}$ atom/cc silicon wafer. [3]

\section{HEATER MODEL}

With a heater design selected, the geometry (shown in figure 3) needs to be optimized to yield the highest heater effectiveness while maintaining the lowest pressure drop. Heat transfer in this instance is a convective transport problem governed by the bulk motion of the fluid. Fin spacing $\left(w_{g a p}\right)$, the height of the channel $\left(h_{o}\right)$, the length of the channel $(L)$, and the number of channels $(N)$ form the heater design space. The dissipated power is driven by the fin width, and the silicon resistivity. The operational parameters of the device are the mass flow rate $(\dot{m})$, the current $(i)$, and the gas supply temperature $\left(T_{f}\right)$. With these parameters specified, a numerical simulation is used to model the steady-state operating conditions of the power dissipated, the pressure drop across the fins, and the exit temperature. From this, the effectiveness can be computed.

A 1-D model (along the heater length) is used and illustrated in figure 3. Variations at each local cross-section are neglected (valid for moderate fin spacing as in the Graetz problem derived in White [4]). By summing the energy inputs into each control volume and non-dimensionalizing, the following system results:

$$
\begin{aligned}
& \frac{d^{2} T_{w}}{d x^{2}}+B i\left(T_{w}-T_{f}\right)-s^{*}=0 \\
& \frac{d T_{f}}{d x}=S t\left(T_{w}-T_{f}\right)
\end{aligned}
$$

where

$$
B i=\frac{h P h_{o}^{2}}{k_{w} A_{c}} ; \quad s^{*}=\frac{\dot{q} h_{o}^{2}}{T_{i} k_{w}} ; \quad S t=\frac{h h_{o} P N}{\dot{m} c_{p}}
$$
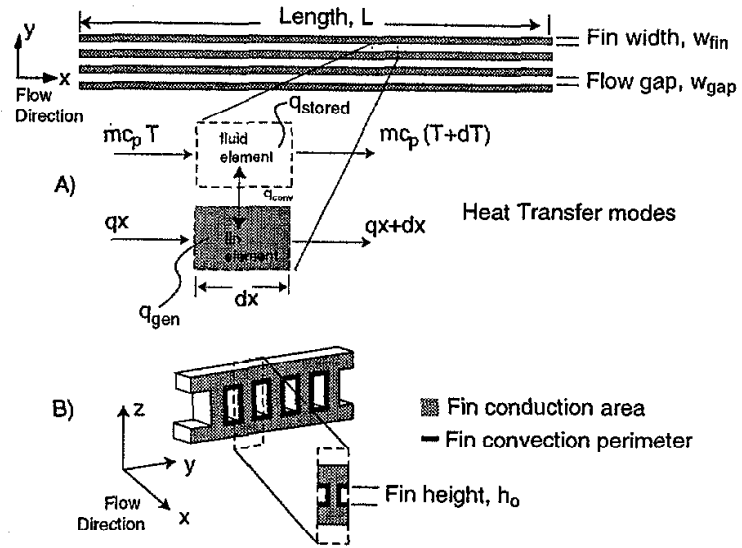

Figure 3. Design simulations are based on a lumped-parameter model that considers the heat generation and transfer along a symmetrical channel element.

The model assumes that heat is generated at a rate set by the electrical model, which is a constant current supply, and temperature-dependant heater resistance depicted in figure 2. The dissipation is treated as a volumetric heating source within the fin $(\dot{q})$. The heat transfer is computed by performing an energy balance on differential fin and fluid elements as shown in figure 3a. The heat is transferred to the fluid convectively and into the up and downstream portions of the fin through conduction (equation 3a). The heat is received by the fluid from the fin and advected downstream (equation $3 b$ ). In order to enhance the fidelity of the model, and to take advantage of the heater symmetry, the analysis is performed for the fin segment shown in figure $3 \mathrm{~b}$. The channel symmetry plane is the boundary one-half of the channel width on each sides of the fin. The conduction area is the full cross-section, and the convection area is the wetted perimeter $(P)$ outlined. By taking into account the upper and lower surfaces, a closer approximation to the heater geometry is achieved. The heat transfer is governed by the fluid mechanics in the channel. Thus, the convection parameter $h$ is a function of Reynolds number and the distance along the fin. The correlation for computing the heat transfer coefficient was developed by Stephan and is reported by Kakac et al. [5] for developing flow in a two-dimensional duct.

The coupled system requires three boundary conditions. The fins are assumed to be insulated at each end $(\mathrm{dT} / \mathrm{dx}=0)$, and the inlet fluid temperature $\left(T_{i}\right)$ is specified $(300 \mathrm{~K})$.

As denoted by their symbols, the coefficient of the wall-fluid temperature difference in the fin equation is a form of the Biot $(B i)$ number, and in the fluid equation the coefficient of the wall-fluid temperature difference is a form of the Stanton $(S t)$ number. The non-dimensional source term is represented by $s^{*}$. It is important to observe the relevance of each of the parameters. The Biot number governs the ratio of the conduction along the fin to the convective heat transfer. High Biot numbers combined with high fin length-to-width aspect ratios result in large temperature differences between the entrance region and exit region of the fins. Because of the high heat transfer in the entrance region, all of the heat generated locally enters into the fluid, and heat generated downstream is conducted along the fin, so it can enter the fluid where gradients are highest. The Stanton number is similar, as it governs the convection to advection ratio along the fin. Thus, when Biot number is low and Stanton number is high, the fins are nearly isothermal. Finally, the source term merely scales the volumetric heat dissipation, relative to that conducted along the wall. When the ratio of Stanton number to the source term is high, then the heater effectiveness will be high. This is due to a majority 
of the dissipated heat being convected into the fluid, which is indicative of a high Stanton number flow.

\section{DESIGN STUDIES}

In order to minimize the pressure drop and maximize heater effectiveness, a number of test cases were explored. To best understand the effect of the governing non-dimensional parameters, we consider four cases and determine how they affect the heater performance. All cases are operating at a mass flow rate of $378 \mathrm{sccms}$, and have 50 micron wide fins. This flow rate was chosen to keep the operating power below 10 watts, and maintain as high a Reynolds number through the nozzle as possible to minimize viscous losses (550 at this condition, which has provided reasonable performance [6]). The heater design and performance parameters are summarized in table 1.

Table 1. Heater Design Cases

\begin{tabular}{|l|l|l|l|l|l|l|l|l|}
\hline Case & $\begin{array}{l}\text { \# of } \\
\text { Fins }\end{array}$ & $\begin{array}{l}\mathrm{L} \\
(\mu \mathrm{m})\end{array}$ & $\begin{array}{l}\mathrm{W} \\
(\mu \mathrm{m})\end{array}$ & $\boldsymbol{\varepsilon}$ & $\begin{array}{l}\Delta \mathrm{P} \\
(\mathrm{atm})\end{array}$ & $\mathrm{Bi}$ & $\mathrm{S}^{*}$ & $\mathrm{St}$ \\
\hline 1 & 5 & 500 & 100 & 99 & 0.21 & 1.1 & 0.5 & 6.5 \\
\hline $\mathbf{2}$ & $\mathbf{1 0}$ & $\mathbf{1 2 5}$ & $\mathbf{5 0}$ & $\mathbf{9 7}$ & $\mathbf{0 . 0 2}$ & $\mathbf{1 . 8}$ & $\mathbf{1 . 5}$ & $\mathbf{1 3 . 0}$ \\
\hline 3 & 5 & 125 & 50 & 85 & 0.04 & 1.8 & 2.5 & 6.6 \\
\hline 4 & 1 & 7000 & 50 & 90 & 0.01 & 0.9 & 1.5 & 0.15 \\
\hline
\end{tabular}

Case 1 is a reasonable design. The pressure drop is $7 \%$ of the total, and a very large temperature rise is achieved. In case 2 , the ratio of the Stanton number to source term has decreased, and as expected, the effectiveness has dropped, but only by $2 \%$. However, the pressure drop has decreased by a factor of 10 . Thus, this is a better design due to the higher injected pressure. For all cases, the dissipated power is 7.7 Watts, which is set by the flow rate and temperature rise. The temperature profiles along the fins are depicted in figure 4 , and the silicon is almost isothermal.

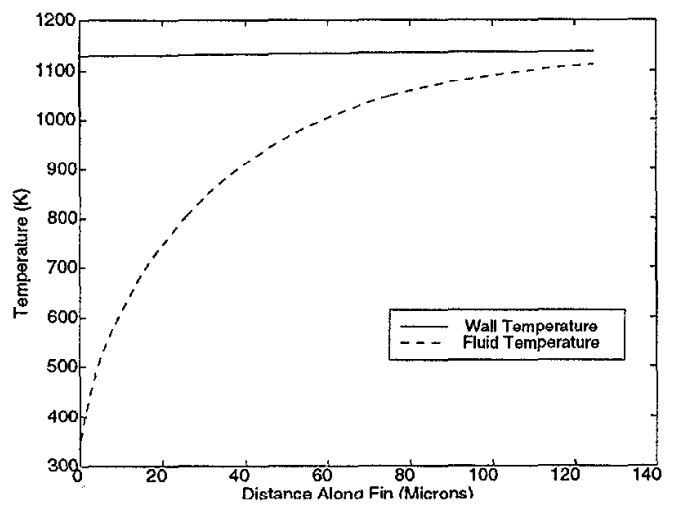

Figure 4. Temperature profile along the channel. Wall temperature is effectively constant due to high conductivity.

Case 3 is the samc as Casc 2, but with 5 fins. By halving the number of fins, the mass flow through a given channel is doubled. This reduces the temperature rise, as well as, increases the pressure drop. This is the lowest Stanton number to source term ratio, and the lowest effectiveness, yet.

Finally, the case representative of a metalized snaking resistor (a flat plate heater) is analyzed as a case with 1 fin (split between the sidewalls). Even with a chamber $7 \mathrm{~mm}$ long, the heater effectiveness is only $90 \%$. By using the design with fins, a more compact arrangement can be attained with a similar pressure drop.

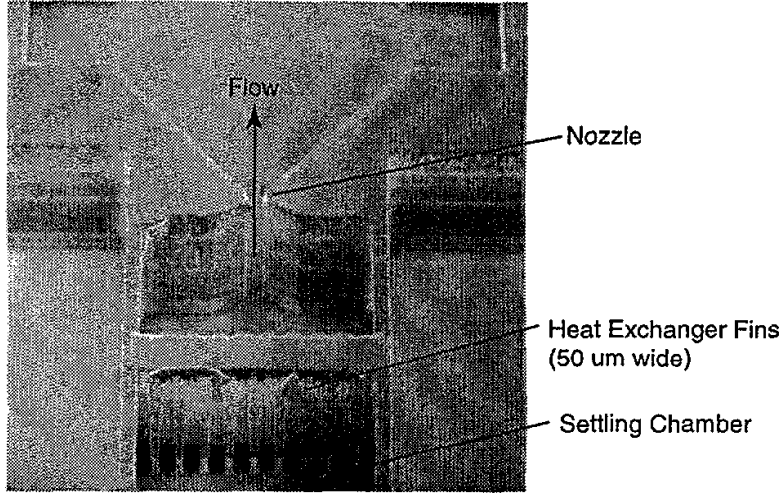

Figure 5. SEM of thruster with integrated heater after first bonding is complete. Bus bar is holding fins together.

\section{HEATER/THRUSTER FABRICATION}

The heater/nozzle system is fabricated in silicon using DRIE. A halo mask is used to simultaneously outline the large cavities as well as define the small heater passages and nozzle throat. By matching these widths, a constant loading is maintained during the etch. In addition, a nested mask is used which allows the throughwafer etch to proceed ahead of the heater-fins. This results in the heater fins being connected by a 50-micron high "bus-bar", which maintains their mechanical integrity and provides electrical functionality. After etching, the cleared flow channels are encapsulated by fusion bonding silicon wafers to the upper and lowers surfaces. Figure 5 is a SEM of the 8.25:1 expansion ratio nozzle with a throat width of 65 microns, a nozzle depth of 491 microns, and the heater analyzed in case 2 of the previous section.

Current is supplied to the heater through electrodes clamped to the top and bottom of the die (see figure 1). The large etched regions on either side of the flow chamber reduce parasitic current paths through the bulk silicon, and direct a larger fraction of the total current through the fins, which are in contact with the flow. The working fluid (Nitrogen) is delivered through Kovar tubing brazed to a stainless steel plate and fixed to the silicon via a glass frit. The plate is clamped with an O-ring to a supply manifold. Further fabrication and assembly details are found in Bayt [2].

\section{EXPERIMENTAL RESULTS}

Figure 6 shows the temperature and power with time for a heater operated with no gas flow. The current is increased in a series of discrete steps, as indicated by the dotted line.

The temperature increases at about $25^{\circ} \mathrm{C} / \mathrm{sec}$ immediately after power is applied, and then asymptotes to a steady value during the next 300 seconds. This is due to the increasing power dissipation with temperature. At a current of $6.06 \mathrm{Amps}$, sufficient power was dissipated to reach the intrinsic temperature $\left(\sim 700^{\circ} \mathrm{C}\right)$. For subsequent times the temperature is seen to rise briefly above the intrinsic temperature before dropping back down due to the inherent stability of the heater architecture. This behavior is observed independently of the driving current, which was continually increased. 


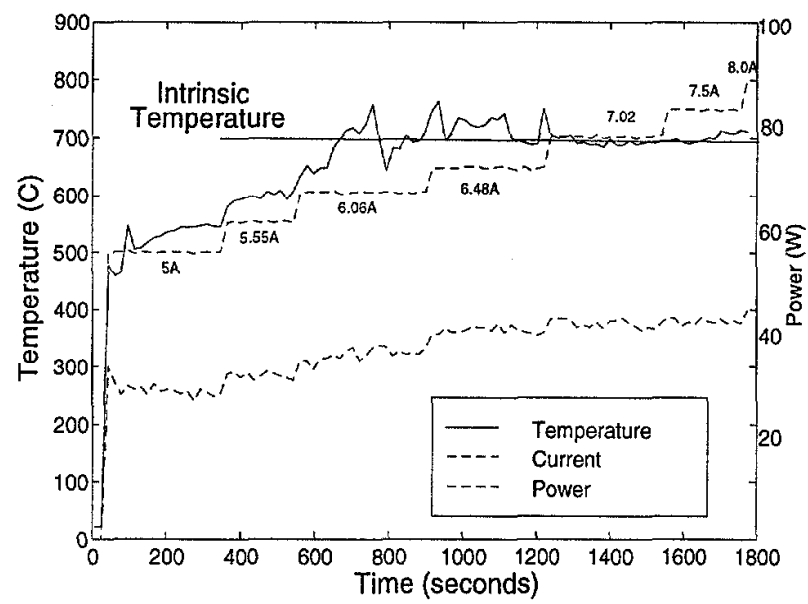

Figure 6. Heater temperature variation with current. $700^{\circ} \mathrm{C}$ intrinsic point is observed, as predicted by the doping level.

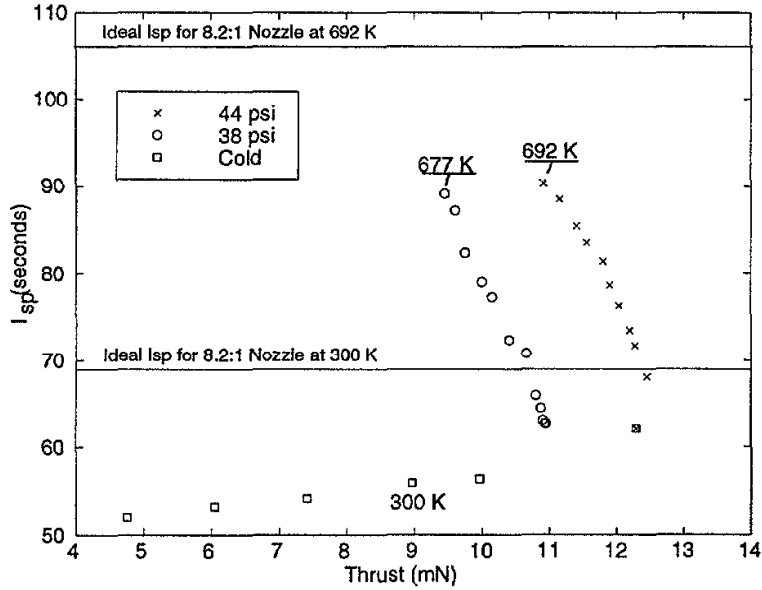

Figure 7. Thrust per unit weight flow $\left(I_{s p}\right)$ is an indication of fuel economy, and thrust indicates propulsive authority.

Thrust tests were performed with the thruster depicted in figure 5 to determine thrust and propellant efficiency. The thrust stand was calibrated using known trial weights and had an accuracy of $\pm 0.5 \mathrm{mN}$. The entire apparatus was housed in a large vacuum chamber with a background pressure of $50 \mathrm{mTorr}$.

Figure 7 shows the specific impulse versus thrust for this system operated over a range of heater powers. 'This illustrates the fuel economy for a given control authority. For fine control, small thrusts are desired, but as shown herc, pcrformance decreases for cold flows. For the cold-flow results (shown by square symbols), the thrust is varied by controlling the operating pressure, and hence the mass flow rate and Reynolds number. For two values of the chamber pressure ( $38 \& 44$ psia), the pressure is held constant and the heater power is varied by increasing the current through the device. As the temperature rises, the specific impulse rises (as expected) and the thrust is observed to decrease. The thrust is composed of contributions from the exit velocity and pressure:

$$
T=\rho U_{e}^{2} A_{e}+P_{e} A_{e}=\frac{P}{R T} \gamma R T M_{e}^{2} A_{e}+P_{e} A_{e}=\dot{m} g I_{s p} .
$$

Note that this predicts that the thrust should be independent of temperature. However, as temperature increases, the Reynolds number decreases, and the exit Mach number $\left(M_{e}\right)$, which ideally is only a function of nozzle geometry, decreases due to the boundary layer blockage effects described in Bayt et al. [6]. For this reason, the observed thrust reduces with temperature, and even more importantly, the $I_{s p}$ relative to the theoretical also decreases significantly. This causes the deviation of the experimental data points in Figure 7 from a vertical line, which would be the ideal for a constant pressure.

Though the $I_{s p}$ is less than the theoretical value, it is $50 \%$ larger than that achieved with the cold gas flow. Thus the spacecraft designer trades electrical power for propellant efficiency, which is desirable since power can be replenished via the solar arrays, whereas propellant cannot be re-supplied.

The overall propulsive efficiency (thrust for a given propellant and electrical power) of this demonstration device is quite low (18\%) primarily due to parasitic electrical losses in the electrical leads and test structure. However, with optimization of the design and better thermal insulation in the packaging, the efficiency could potentially be raised to $40 \%$.

\section{CONCLUSIONS}

The present design has two key features that make it attractive for integrated micro-heat exchangers. First, the use of bulk silicon as both the structural and electrical material simplifies the system architecture and allows for high fluid/heater contact area. Second, the properties of silicon at the intrinsic point are very attractive for stable operation, particularly for gases where thermal runaway can be problematic. By increasing the chamber energy in a microthruster, the mass flow required for a given thrust level is greatly reduced. This translates into increased satellite life for a given propellant supply. Although the device efficiency is low in this initial example, little attempt has been made to minimize parasitic losses through the leads and test structure and one expects the efficiency could be improved in future designs.

\section{ACKNOWLEDGEMENTS}

The authors would like to thank Professor. Martin Schmidt of the MIT Microsystem Technology Lab who first suggested the exploitation of the intrinsic properties of silicon for the heater. His guidance was invaluable in the design and fabrication phases. Additional fabrication support was provided by Dr. Arturo Ayon. This work was supported by the Air Force Office of Scientific Research.

\section{REFERENCES}

1. S.W. Janson, "Batch-Fabricated Resistojets: Initial Results". In International Electric Propulsion Conference. IEPC-97-070, 1997.

2. R. L. Bayt Analysis, Fabrication and Testing of a MEMS-based Micropropulsion System Ph.D. Thesis, M.I.T., 1999.

3. G. L. Pearson, and J. Bardeen, "Electrical Properties of Pure Silicon and Silicon Alloys Containing Boron and Phosphorous", Physical Review, 75, 1 (1949).

4. F.M. White, Viscous Fluid Flow, $2^{\text {nd }}$ Edition, 1991.

5. R.K. Kakac, S. Shah and A.E. Bergles. Low Reynolds Number Heat Exchangers. Hemisphere Publishing, $1^{\text {st }}$ edition, 1983.

6. R. L. Bayt, K. S. Breuer, and A. A. Ayon "DRIE-Fabricated Nozzles for Generating Supersonic Flows in Micropropulsion Systems", Technical Digest of the 1998 Solid-State Sensor and Actuator Workshop, Hilton Head Isl., SC, 6/8-11/98, Transducer Research Foundation, Cleveland (1998), pp. 312 - 316. 